\begin{tabular}{lc|c}
\hline Bentham OPEN & The Open Nursing Journal & $\begin{array}{l}\text { The } \\
\text { Open Nursing } \\
\text { lournal } \\
\text { CrossMark }\end{array}$ \\
\hline
\end{tabular}

\title{
The Use of Phone Technology in Outpatient Populations: A Systematic Review
}

\author{
Ana C. Duarte ${ }^{*}$ and Sue A. Thomas \\ Department of Family and Community Health, University of Maryland School of Nursing, Baltimore, MD 21201, USA
}

Received: March 20, 2015

Revised: June 02,2015

Accepted: June 15, 2015

\section{Abstract:}

Objective:

A systematic review was conducted to identify the types of phone technology used in the adult outpatient population with a focus on Hispanic patients and psychiatric populations.

\section{Methods:}

A search for articles was conducted on the EMBASE, PubMed and PsycINFO databases. Articles reviewed were peer-reviewed, fulltext, English language and published through mid-November 2014.

\section{Results:}

Twenty-one articles were included in this review and grouped according to combinations of phone technology, medical specialty area and population. For all articles, phone technology was defined as telephone, cell, or smart phone. Technology was used in psychiatry with Hispanic population in four articles, in psychiatry with non-Hispanic population in seven articles and in other specialties with Hispanic population in ten articles. Articles were evaluated for quality. Six articles were assessed as strong, eight were moderate and seven were weak in global quality. Interventions included direct communication, text messaging, interactive voice response, camera and smart phone app. Studies with Hispanic populations used more text messaging, while studies in psychiatry favored direct communication. The majority of articles in all groups yielded improvements in health outcomes.

\section{Conclusion:}

Few studies have been conducted using phone technology in Hispanic and psychiatric populations. Various phone technologies can be helpful to patients in diverse populations and have demonstrated success in improving a variety of specific and overall healthcare outcomes. Phone technologies are easily adapted to numerous settings and populations and are valuable tools in efforts to increase access to care.

Keywords: Adherence, Hispanic, Patient outcomes, Psychiatry, Self-care management, Technology.

\section{INTRODUCTION}

Alexander Graham Bell's invention, the telephone, fundamentally transformed person-to-person communications, allowing efficient and immediate voice contact between two remote parties [1]. In the almost 140 years since its introduction, this communications technology has experienced an explosive evolution. Mobile phones and personal digital assistants (PDAs) have been widely available to the public for almost three decades [2]. Wireless technology continued to advance rapidly and the mobile phone's most recent incarnation, the smart phone, has propelled growth in this technology and similar devices to the present day [3]. Like the introduction of computers into households across the

\footnotetext{
* Address correspondence to this author at the Department of Family and Community Health, University of Maryland School of Nursing, 655 W. Lombard Street, Room 592, Baltimore, MD 21201, USA; Tel: 410-706-3047; Fax: 410-706-0253; E-mail: aduarte@son.umaryland.edu
} 
globe, the evolution of mobile technology has changed the way people communicate and access information.

The term Digital Divide refers to inequalities in access to internet. While this situation may have been the case in the past, this gap has been shrinking at a very rapid pace. Year-end estimates for 2014 worldwide cell phone subscriptions in developed countries were expected to near 1.5 billion, which translates to approximately 121 subscriptions per 100 inhabitants [4]. Today, cell phone ownership among Americans is at 91\%, minority groups are more likely to use a smart phone for internet access, and Blacks, whites, and Hispanics are equally likely to own mobile phones $[5,6]$. Additionally, $80 \%$ of smart phone owners surveyed worldwide by the analytics software company FICO were interested in using their phones for healthcare communications, specifically seeking reminders of upcoming appointments (76\%) as well as reminders to make appointments or take medications (69\%) [7]. A 2013 PEW survey found that $56 \%$ of American adults own a smart phone as opposed to more basic cell phone models, up from $46 \%$ the year before and 35\% two years prior [8]. This growth trends across gender, age, race, and cultural/ethnic demographics. The high rates of ownership and usage of the instruments to access health information on the internet, as well as strong user preferences for healthcare communications via smart phone, suggest that this widespread personal technology can be useful in reducing health disparities and improving access to healthcare via increased communication [7, 9, 10].

Mental health issues are of serious concern and place a great burden on all levels of society. Individuals, families, and entire communities are affected, and access to care is a barrier worldwide. The gaps between mental healthcare demand and supply are estimated at 76\%-85\% in low and middle-income countries and between $35 \%$ and $50 \%$ in highincome countries by the World Health Organization (WHO) [11]. U.S. Healthy People 2020 guidelines identify increasing access to mental health and a focus on prevention as important goals, placing increased demands on both mental and physical healthcare resources [12]. Improved adherence to treatment regimens yields improved patient outcomes. Despite increased demand and attempts to address it, patients continue to have trouble adhering to both appointments and medication regimens resulting in sub-optimal outcomes [13 - 18].

Several reviews have examined the use of cell and voice technology in enhancing self-care and disease management, behavioral changes, preventive care, and appointment attendance in a variety of healthcare settings, age groups, and health conditions with particular progress in the realm of text messaging interventions [19 - 22]. The majority of studies identified in these reviews were conducted in countries other than the U.S. While two Cochrane reviews [21, 22] examined trials in low, middle, and high-income countries, none of the reviewed studies specifically examined racial, ethnic, or socioeconomic minority groups. Likewise, none of the reviews yielded studies targeting phone interventions in the psychiatric specialty area.

The goal of this review is to identify and evaluate the types of phone technology used in the adult outpatient population, focusing on Hispanic patients and psychiatric populations, two areas not reviewed in depth to the authors' knowledge. The results of this review will identify quality of existing studies and gaps in the literature, both of which will bring to light areas for further research. Innovative technological interventions specifically directed toward these populations, which are vulnerable due to health disparities or the stigma of illness, may lead to improved appointment and medication adherence and yield improved health management and outcomes.

\section{METHODS}

\section{Design}

A systematic review was conducted using the guidelines of the Preferred Reporting Items for Systematic Reviews and Meta-Analyses (PRISMA) Statement [23, 24]. Relevant articles were identified and selected for ultimate review, synthesis, and grading of the evidence. Because studies were expected to be of diverse types, settings, comparators and outcomes, this systematic review did not lend itself to the additional step of conducting a meta-analysis [25].

\section{Literature Search}

The PRISMA Statement $[23,24]$ also guided the literature search. A priori criteria for inclusion yielded studies that were peer-reviewed, full-text, English language, conducted in any country and involved human subjects over the age of 18. Inclusion criteria used phone technology intervention with either a psychiatric and/or Hispanic population. Specifically excluded were review articles, meta-analyses, case studies, as well as dissertations and poster presentations. Systematic searches were conducted on three electronic databases, EMBASE, PubMed, and PsycINFO (EBSCO). An initial search was performed in early March 2013 for all articles to date; because technology use in healthcare is relatively new, no lower date parameter was used to exclude older studies initially, though during full-text screening, 
articles published before 2003 were eliminated. A second search was conducted in early November 2014 to include new articles since the first search. Google Scholar and hand searches of full-article reference lists were also performed with each series of electronic database searches.

Searches were conducted using various combinations of terms in the following broad categories: psychiatry, adherence, technology, and Hispanic. Specific search terms have been summarized in Table $\mathbf{1}$.

Two reviewers independently followed a three-step screening process to narrow down the list of studies for final evaluation in this systematic review: (i) screening by title; (ii) screening by abstract; and (iii) screening of full-text articles. Prior to moving forward, consensus was achieved at each step.

\section{Data Extraction}

Data extraction was performed by one reviewer who created three detailed evidence tables with the following information from each article: author, year, location, study design, sample size, population, setting, technology and intervention, comparators and main outcomes. Both reviewers confirmed accuracy of table data by re-checking against the original articles. Any data discrepancies were discussed and appropriate changes or corrections made. An additional column was added to each evidence table reflecting global quality rating.

\section{Quality Assessment}

Quality of the studies was evaluated using the Qualitative Assessment Tool for Quantitative Studies (QATQS) [26, 27]. Validity and reliability, in randomized as well as non-randomized studies, have been demonstrated for the QATQS $[27,28]$. Eight components of quality are examined by the QATQS: (i) selection bias, (ii) design, (iii) confounders, (iv) blinding, (v) data collection, (vi) withdrawals and drop-outs, (vii) intervention integrity, and (viii) analysis appropriate to question. Ratings of strong, moderate or weak are assigned to each of the first six components, in accordance with the tool's guide and dictionary. These individual ratings are combined to produce a global quality rating (Table 2). Each reviewer performed an independent evaluation, then both came together to discuss discrepancies and arrive at $100 \%$ agreement on final study quality ratings.

\section{Article Evaluation}

Each article was analyzed in the context of technology used, population and medical specialty area. Three detailed evidence tables were created, grouping articles by phone technology, population, and specialty and including summarized information from each article, as well as quality rating per the QATQS [26].

\section{RESULTS}

Initial literature searches yielded 1, 757 articles from the databases and hand searches yielded an additional 22 articles (Fig. 1). Fifty articles were duplicates, leaving 1, 729 articles to be screen by title and abstract. Of these, 1, 384 were excluded on title and 176 were excluded on abstract. After full-text assessment of 169 articles, 21 were retained in the final list for qualitative synthesis [29 - 49]. The articles were grouped as follows for analysis: (i) four articles using phone technology in psychiatry with Hispanic populations [34, 36, 40, 49], (ii) seven articles using phone technology in psychiatry with non-Hispanic populations [29, 35, 37 - 39, 43, 47], and (iii) ten articles using phone technology in other specialties with Hispanic populations [30 - 33, 41, 42, 44 - 46, 48]. For all articles, phone technology was defined as telephone, cell, or smart phone. The term psychiatry encompassed articles specific to the field, as well as articles in other disciplines that targeted psychiatry-specific outcomes. Of the 21 studies, ten were randomized controlled studies (RCTs) [29 - 31, 33, 34, 40, 41, 46 - 48], nine were cohort studies [32, 35 - 38, 42, 44, 45, 49] and two were controlled clinical trials (CCTs) [39, 43]. With one exception [29], all articles reported on studies conducted in the United States.

\section{Technology Use in Psychiatry with Hispanic Populations}

Four articles (two RCTs and two cohort studies) [34, 36, 40, 49] described interventions using phone technology in Hispanic populations with outcomes specifically in the realm of psychiatry. Details regarding these studies can be found in Table 3. A total of 2, 205 participants were included in the studies, the majority in safety net populations [40, 49] and the remainder in the general population $[34,36]$. Two articles $[34,40]$ were of strong quality while one each were found to be of moderate [36] and weak [49] quality [26]. 
Table 1. Summary of systematic search terms by general category.

\begin{tabular}{|l|l|}
\hline General Category & \multicolumn{1}{|c|}{ Search Terms } \\
\hline Psychiatry & mental disorders, mental health, mental health services, psychiatric \\
\hline Adherence & $\begin{array}{l}\text { adherence, appointment adherence, appointment compliance, compliance, follow up, medication adherence, medication } \\
\text { compliance, medication follow up, medication management, patient adherence, patient compliance, self care, self management, } \\
\text { treatment adherence, treatment compliance }\end{array}$ \\
\hline Technology & $\begin{array}{l}\text { applications, apps, interactive, internet, mhealth, mms, mobile health, multimedia, multi media, multimedia messag*, short } \\
\text { messag*, sms, social media, tablet*, texted, texting, cell phone, cellular phone, computer assisted, internet based, mobile phone, } \\
\text { online, smartphone, smart phone, telecommunications, telemedicine, telephone, text messag*, wireless phone, wireless telephone }\end{array}$ \\
\hline Hispanic & $\begin{array}{l}\text { Central American, Cuban, Hispanic American, Latin American, Mexican, Puerto Rican, Salvadoran, Hispanic*, Latino*, } \\
\text { Spanish speaking }\end{array}$ \\
\hline
\end{tabular}

Table 2. QATQS assessment and grading criteria.

\begin{tabular}{|c|c|}
\hline Quality Component & Assessment Criteria \\
\hline Selection Bias & $\begin{array}{l}\text { Degree to which participants are representative of target population } \\
\text { Percentage of study candidates who agreed to participate }\end{array}$ \\
\hline Design & $\begin{array}{l}\text { Study design } \\
\text { Description of randomization process } \\
\text { Appropriate randomization sequence }\end{array}$ \\
\hline Confounders & $\begin{array}{l}\text { Controlling of confounders via design } \\
\text { Baseline balance as to confounders }\end{array}$ \\
\hline Blinding & $\begin{array}{l}\text { Outcome assessors } \\
\text { Participants }\end{array}$ \\
\hline Data Collection & Reliability and validity of assessment tools/methods \\
\hline Withdrawals and Drop-Outs & $\begin{array}{l}\text { Reporting of withdrawal numbers and reasons } \\
\text { Percentage of participants completing the study }\end{array}$ \\
\hline Intervention Integrity & $\begin{array}{l}\text { Percentage of participants receiving intended intervention } \\
\text { Measurement of consistency of intervention administration } \\
\text { Contamination/unintended intervention }\end{array}$ \\
\hline Analysis Appropriate to Question & $\begin{array}{l}\text { Level of allocation and analyses } \\
\text { Appropriateness of statistical methods to study design } \\
\text { Analyses for intention-to-treat }\end{array}$ \\
\hline Global Quality Rating & $\begin{array}{l}\text { Strong }=4 \text { strong ratings and } 0 \text { weak ratings } \\
\text { Moderate }=\text { less than } 4 \text { strong ratings and } 1 \text { weak rating } \\
\text { Weak }=2+\text { weak ratings }\end{array}$ \\
\hline
\end{tabular}

All four articles used phone technology interventions for mental health outcomes, though none specified the type of phone used in the intervention. Two articles described studies in breast cancer survivors; one used telephone psychoeducation to address depression in this population [34] and the other used therapy and cancer education to help with more general mental health support [36]. The other two articles addressed studies using phone technology for depression in individuals with diabetes; the first provided collaborative care using the telephone [40] and the second used a telephone interactive voice response (IVR) system [49]. Two articles [34, 40] reported improvement in depressive symptoms. Additionally, one of these articles [40] noted ongoing depression treatment and overall improvement in quality of life, but did not report significant differences in clinical outcomes for diabetes as a result of the intervention. A third article reported significant improvements in psychological, physical, social, and spiritual QOL [36]. The remaining article [49] did not report any outcomes as it described a study currently underway.

\section{Technology Use in Psychiatry with Non-Hispanic Populations}

Phone technology interventions in non-Hispanic populations targeting outcomes in the area of psychiatry are described in seven articles (two RCTs, two CCTs, and three cohort studies) [29, 35, 37 - 39, 43, 47]. Study details are described in Table 4. These studies included a total of 45, 767 participants. Four studies included subjects from the general population [35, 38, 39, 43], two studies involved people who had completed inpatient programs [29, 47] and one study recruited from a federally qualified health center (FQHC) [37]. Two articles were rated strong in quality [29, 47], two were rated moderate [37, 39], and the remaining three were rated weak [35, 38, 43].

Three articles described the use of cell or mobile phone interventions [29, 37, 43] while the remaining four did not specify the type of phone used in the intervention [35, 38, 39, 47]. The three articles using mobile phone interventions 


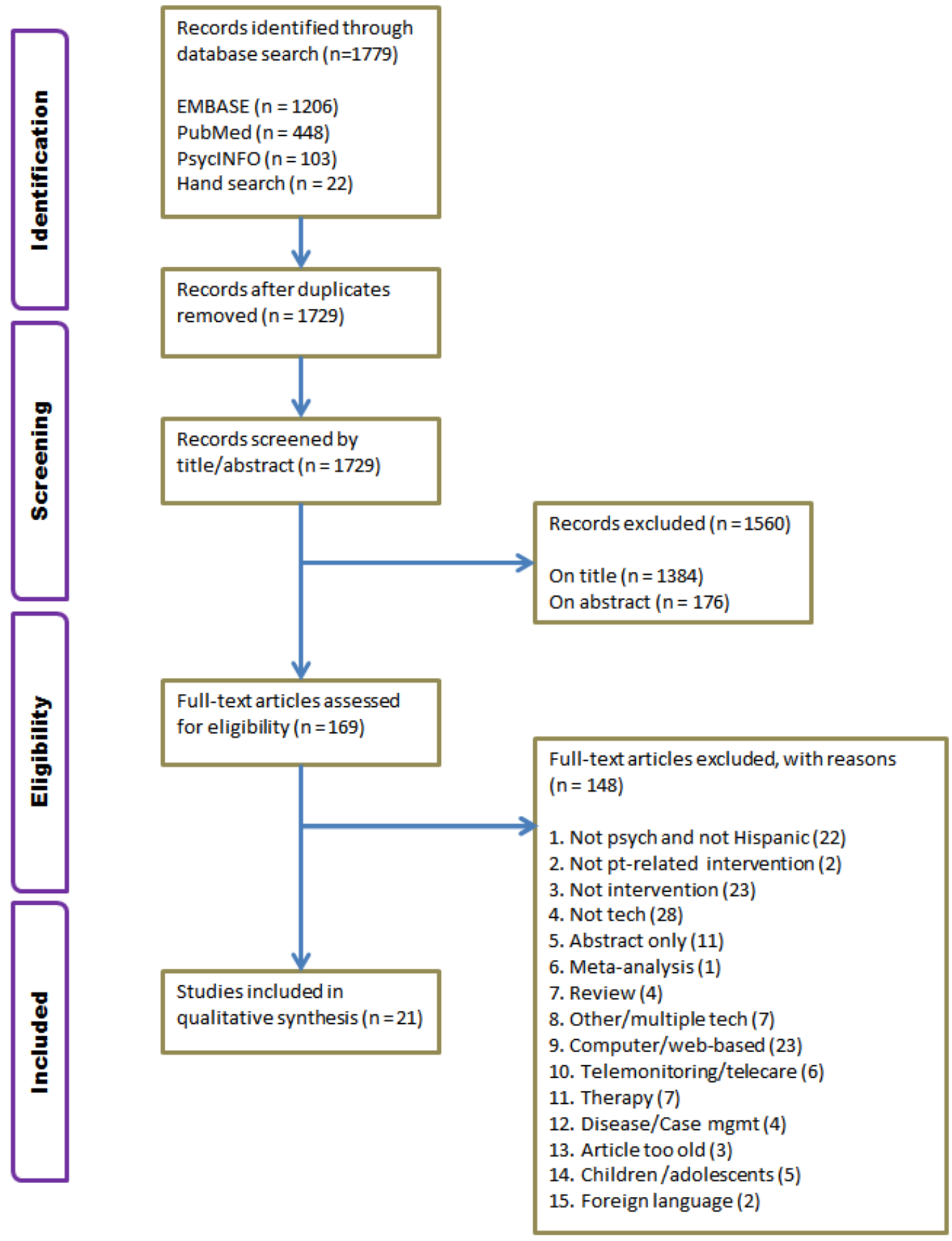

Fig. (1). Flow diagram for literature search.

all did so in participants with substance abuse problems. One pilot study conducted in Ireland used a text messaging intervention in a group of patients diagnosed with depression and alcohol abuse [29]. Another pilot used IVR in the dually-diagnosed homeless population [37]. The last of these articles used cell phone cameras to take pictures of morning medications of methamphetamine-dependent individuals [43]. Of the remaining four articles, three involved counseling or support call interventions for patients with depression [35], serious and persistent mental illness (SPMI) [39] and posttraumatic stress disorder (PTSD) in veterans [47], while one used IVR technology in depression [38]. Of five articles addressing medication adherence, four reported improvements or high levels of adherence [35, 37, 39, 43] and one reported no significant impact to adherence, though age was identified as a confounder [38]. In addition, one 
study reported greater likelihood of therapy continuation, improvements in refill timeliness, symptom burden and severity, and mental quality of life (QOL) with no improvements in physical QOL [35]. Other studies reported high ability to reach participants using technology and subjective improvements in communications [37], as well as decreased emergency department (ED) utilization [39] and usefulness of the technology in measuring adherence [43].

Table 3. Articles that included technology use in psychiatry with Hispanic populations.

\begin{tabular}{|c|c|c|c|c|c|}
\hline $\begin{array}{c}\text { First } \\
\text { Author, } \\
\text { Year } \\
\text { Location } \\
\text { Study } \\
\text { Design }\end{array}$ & $\begin{array}{c}\text { Sample Sizes/ } \\
\text { Population/Setting }\end{array}$ & Technology/Intervention & Comparators & $\begin{array}{c}\text { Main Outcomes Intervention v. } \\
\text { Comparators }\end{array}$ & $\begin{array}{l}\text { Global } \\
\text { Quality } \\
\text { Rating }\end{array}$ \\
\hline $\begin{array}{l}\text { Ashing, } \\
2014 \text { [34] } \\
\text { USA, CA } \\
\text { RCT }\end{array}$ & $\begin{array}{l}\mathrm{n}=252 / \text { Randomized } \mathrm{n}=221 \\
(\mathrm{I}: \mathrm{n}=110,99 \text { completed, C: } \\
\mathrm{n}=111,100 \text { completed }) ; 18+ \\
\text { y.o., English or Spanish } \\
\text { speaking Latina BCA } \\
\text { survivors via } \text { CA Cancer } \\
\text { Registry; general population }\end{array}$ & $\begin{array}{l}\text { Telephone. Eight bi-weekly psycho- } \\
\text { educational interventions, } 40-50 \\
\text { minutes plus survivorship booklet. }\end{array}$ & $\begin{array}{l}\text { Survivorship booklet } \\
\text { only. }\end{array}$ & $\begin{array}{l}\text { Level of depressive symptoms. } \\
\text { Over time, within language } \\
\text { group, significantly decreased. } \\
\text { Follow-up also showed } \\
\text { significant decrease in symptoms. } \\
\text { By language preference, both } \\
\text { English and Spanish language- } \\
\text { preferred showed significant } \\
\text { decrease from baseline to follow- } \\
\text { up. }\end{array}$ & Strong \\
\hline $\begin{array}{l}\text { Badger, } \\
2013[36] \\
\text { USA, AZ } \\
\text { Cohort }\end{array}$ & $\begin{array}{l}\mathrm{n}=80 \text { dyads ( } 160 \text { total) (I: } \\
\mathrm{n}=40, \mathrm{C}: \mathrm{n}=40), \text { Latina BCA } \\
\text { survivors and their } \\
\text { supportive partners; general } \\
\text { population }\end{array}$ & $\begin{array}{l}\text { Telephone. (TIP-C intervention) } \\
\text { Weekly interpersonal psychotherapy } \\
+ \text { CA education for BCA survivors, } \\
\text { every other week for supportive } \\
\text { partners. English or Spanish. }\end{array}$ & $\begin{array}{l}\text { (THE intervention) } \\
\text { Standardized } \\
\text { educational materials } \\
\text { sent prior to } \\
\text { intervention, reviewed } \\
\text { over the phone. Weekly } \\
\text { sessions for BCA } \\
\text { survivors, every other } \\
\text { week for supportive } \\
\text { partners. English or } \\
\text { Spanish. }\end{array}$ & $\begin{array}{l}\text { Psychological, physical, social, } \\
\text { and spiritual QOL. Significant } \\
\text { improvements (in all EXCEPT } \\
\text { spiritual) for BCA survivors in } \\
\text { both groups over time. } \\
\text { Significant improvements (in } \\
\text { ALL QOL areas) for supportive } \\
\text { partners in both groups over time. } \\
\text { No clear support demonstrating } \\
\text { one intervention better than the } \\
\text { other. }\end{array}$ & Moderate \\
\hline $\begin{array}{l}\text { Ell, } 2011 \\
{[40]} \\
\text { USA, CA } \\
\text { RCT }\end{array}$ & $\begin{array}{l}\text { n=387 (I: n=193, } 138 @ 24 \\
\text { mos; C: n=194, 126@24 } \\
\text { mos) low-income adults } \\
\text { with diabetes \& depression - } \\
\text { part of Multifaceted } \\
\text { Diabetes and Depression } \\
\text { Program (MDDP); English } \\
\text { or Spanish speaking, in } \\
\text { primary safety net care }\end{array}$ & $\begin{array}{l}\text { Telephone. Educational pamphlets } \\
\text { and resource lists plus } \\
\text { socioculturally adapted collaborative } \\
\text { care for depression in primary care } \\
\text { (psychotherapy, antidepressants, or } \\
\text { both; telephone symptom } \\
\text { monitoring \& relapse prevention) }\end{array}$ & $\begin{array}{l}\text { Enhanced Usual Care } \\
\text { includes same } \\
\text { pamphlets and resource } \\
\text { lists as intervention } \\
\text { group. PCPs also could } \\
\text { Rx antidepressants and } \\
\text { provide counseling or } \\
\text { refer for community } \\
\text { mental health. } \\
\end{array}$ & $\begin{array}{l}\text { Depression care. More going Tx } \\
\text { at } 24 \text { months; Depression } \\
\text { symptoms. Improved at } 24 \\
\text { month; QOL. Overall } \\
\text { improvement, narrowing over } \\
\text { time, not significant at } 24 \text { mos; } \\
\text { DM clinical outcomes. No } \\
\text { significant differences. }\end{array}$ & Strong \\
\hline $\begin{array}{l}\text { Wu, } 2014 \\
\text { [49] } \\
\text { USA, CA } \\
\text { Cohort }\end{array}$ & $\begin{array}{l}\mathrm{n}=1406(\mathrm{I}: \mathrm{n}=442, \mathrm{C} 1: \\
\mathrm{n}=484, \mathrm{C} 2: \mathrm{n}=480) \text { low- } \\
\text { income, predominantly } \\
\text { Hispanic/Latino adults with } \\
\text { diabetes, English or Spanish } \\
\text { speaking, county safety net } \\
\text { clinics }\end{array}$ & $\begin{array}{l}\text { Telephone IVR. Technology- } \\
\text { facilitated depression care (TC). } \\
\text { Educational/resource materials }+ \\
\text { calls from automated telephonic } \\
\text { assessment (ATA) call system. } \\
\text { Monthly ATA calls for depressed at } \\
\text { baseline, Q } 3 \text { mos. for not depressed } \\
\text { at baseline. TC group also gets } \\
\text { telephone appt reminders. }\end{array}$ & $\begin{array}{l}\text { In addition to } \\
\text { educational and } \\
\text { resource materials: } 1 \text { ) } \\
\text { Usual care (UC), } \\
\text { traditional clinic } \\
\text { depression and diabetes } \\
\text { care; } 2 \text { ) Collaborative } \\
\text { care team supported } \\
\text { care (SC) includes RN, } \\
\mathrm{NP}, \mathrm{SW} \text { to assist with } \\
\text { MH issues for } 6 \text { mos, } \\
\text { then return to usual } \\
\text { care. }\end{array}$ & $\begin{array}{l}\text { Depression outcomes. Treatment } \\
\text { adherence. Social and economic } \\
\text { stress reduction. DM self-care } \\
\text { mgmt. Health care utilization. } \\
\text { Care mgmt. model cost. Cost- } \\
\text { effectiveness. Comparisons. No } \\
\text { results; project in progress. Goal: } \\
\text { reduce health disparities via } \\
\text { improved outcomes and reduced } \\
\text { costs. }\end{array}$ & Weak \\
\hline
\end{tabular}

Abbreviations: $\mathrm{RCT}=$ randomized controlled trial, $\mathrm{I}=$ intervention, $\mathrm{C}=$ comparator, y.o.=years old, $\mathrm{BCA}=$ breast cancer, $\mathrm{CA}=$ cancer, $\mathrm{TIP}-\mathrm{C}=$ telephone interpersonal counseling, THE=telephone health education, $\mathrm{QOL}=$ quality of life, $\mathrm{mos}=\mathrm{months}, \mathrm{PCPs}=\mathrm{primary}$ care providers, $\mathrm{Rx}=\mathrm{prescribe}$, $\mathrm{Tx}=$ treatment, $\mathrm{DM}=$ diabetes mellitus, $\mathrm{IVR}=$ interactive voice response, appt=appointment, $\mathrm{RN}=$ registered nurse, $\mathrm{NP}=$ nurse practitioner, $\mathrm{SW}=$ social worker, $\mathrm{MH}=$ mental health

The article focusing on depression and alcoholism reported improved depression, abstinence, and functioning, while noting no significant difference in compulsion to drink [29]. Lastly, the study addressing PTSD reported no significant differences in PTSD or depression, aggressive behaviors, alcohol or drug problems, QOL, time to rehospitalization, or engagement in care [47]. 


\section{Technology Use in Other Specialties with Hispanic Populations}

Ten articles fell into the category of phone technology use with Hispanics in other medical specialty areas (six RCTs and four cohort studies) [30 - 33, 41, 42, 44 - 46, 48]. Details of these studies are listed in Table 5. A total of 1, 615 participants were included in these studies. Subjects were recruited from general populations for three studies [30, 44, 45], safety net populations for three studies [31 - 33], FQHC for two studies [42, 48], and one each from county health department [41] and community settings [46]. Using QATQS rating criteria [26], two articles were determined to

Table 4. Articles that included technology use in psychiatry with non-Hispanic populations.

\begin{tabular}{|c|c|c|c|c|c|}
\hline $\begin{array}{l}\text { First } \\
\text { Author, } \\
\text { Year } \\
\text { Location } \\
\text { Study } \\
\text { Design } \\
\end{array}$ & $\begin{array}{c}\text { Sample Sizes/ } \\
\text { Population/Setting }\end{array}$ & Technology/Intervention & Comparators & $\begin{array}{l}\text { Main Outcomes } \\
\text { Intervention v. } \\
\text { Comparators }\end{array}$ & $\begin{array}{l}\text { Global } \\
\text { Quality } \\
\text { Rating }\end{array}$ \\
\hline $\begin{array}{l}\text { Agyapong, } \\
2012 \text { [29] } \\
\text { Ireland } \\
\text { RCT (Pilot) }\end{array}$ & $\begin{array}{l}\mathrm{n}=54(\mathrm{I}: \mathrm{n}=26, \mathrm{C}: \mathrm{n}=28) \text { adult } \mathrm{pts} \\
\mathrm{w} / \text { depression and ETOH; completers } \\
\text { of inpatient program }\end{array}$ & $\begin{array}{l}\text { Mobile phone. BID supportive text } \\
\text { messages. }\end{array}$ & $\begin{array}{l}\text { Thank-you text } \\
\text { messages every } 2 \\
\text { weeks. }\end{array}$ & $\begin{array}{l}\text { Depression and abstinence. } \\
\text { Improved. Functioning. } \\
\text { Improved. Compulsion to } \\
\text { drink. No significant } \\
\text { difference. }\end{array}$ & Strong \\
\hline $\begin{array}{l}\text { Aubert, } \\
2003 \text { [35] } \\
\text { USA } \\
\text { Cohort }\end{array}$ & $\begin{array}{l}\mathrm{n}=5624 \text { (I: } \mathrm{n}=505, \mathrm{C} 1: \mathrm{n}=1375, \mathrm{C} 2: \\
\mathrm{n}=3744) \text { adult insurance pharma plan } \\
\text { members with depression with new } \\
\text { prescription (none in last } 180 \text { days) for } \\
\text { an antidepressant medication; general } \\
\text { population }\end{array}$ & $\begin{array}{l}\text { Telephone. Four telephone } \\
\text { counseling calls. Five educational } \\
\text { mailings. Toll-free number set up } \\
\text { for participant questions. }\end{array}$ & \begin{tabular}{|l|} 
1) Minimal \\
intervention: \\
completed first call \\
but did not consent \\
to continuing \\
interventions. 2) \\
No interventions. \\
\end{tabular} & \begin{tabular}{|l|} 
Medication adherence. \\
Improved. Therapy \\
continuation. More likely. \\
Refill timeliness. Improved. \\
Symptom burden. \\
Improved. QOL-mental. \\
Improved. QOL-physical. \\
No significant difference. \\
Symptom severity. \\
Improved. \\
\end{tabular} & Weak \\
\hline $\begin{array}{l}\text { Burda, 2012 } \\
\text { [37] } \\
\text { USA, MD } \\
\text { Cohort } \\
\text { (Pilot) }\end{array}$ & $\begin{array}{l}\text { n=10 adult homeless dual-Dx'd pts, } \\
\text { mostly black, mostly men; FQHC }\end{array}$ & $\begin{array}{l}\text { Cell phone IVR. Daily phone } \\
\text { calls, two attempts. }\end{array}$ & None & \begin{tabular}{|l|} 
Medication adherence. \\
High levels reported. \\
Ability to reach \\
participants. High. \\
Subjective report of \\
communications. Improved.
\end{tabular} & Moderate \\
\hline $\begin{array}{l}\text { Castle, } 2012 \\
\text { [38] } \\
\text { USA, PA } \\
\text { Cohort }\end{array}$ & $\begin{array}{l}\mathrm{n}=39,020(\mathrm{I} 1: \mathrm{n}=293, \mathrm{I} 2: \mathrm{n}=11,280, \\
\mathrm{I3}: \mathrm{n}=27,447) \text { adult insurance pharma } \\
\text { plan members newly Rx'd } \\
\text { antidepressant medication; general } \\
\text { population }\end{array}$ & $\begin{array}{l}\text { Telephone IVR. Calls to } \\
\text { participants with option to listen } \\
\text { then transfer to depression mgmt } \\
\text { program. If not reached, msg left } \\
\text { with callback number. }\end{array}$ & $\begin{array}{l}3 \text { a posteriori } \\
\text { intervention } \\
\text { groups: 1) reached, } \\
\text { transferred, 2) } \\
\text { reached, not } \\
\text { transferred, 3) not } \\
\text { reached. }\end{array}$ & $\begin{array}{l}\text { Medication adherence. Not } \\
\text { significantly impacted by } \\
\text { intervention. Age was } \\
\text { confounder, as increase in } \\
\text { adherence seen with } \\
\text { increasing age. }\end{array}$ & Weak \\
\hline $\begin{array}{l}\text { Cook, } 2008 \\
{[39]} \\
\text { USA, CO } \\
\text { CCT }\end{array}$ & $\begin{array}{l}\mathrm{n}=202 \text { (I: } \mathrm{n}=51, \mathrm{C}: \mathrm{n}=151) \text { adult } \\
\text { Medicaid members SPMI, received } \\
\text { 2nd gen antipsychotic in last } 30 \text { days; } \\
\text { mostly women; general population }\end{array}$ & $\begin{array}{l}\text { Telephone. Adherence counseling } \\
\text { via } \mathrm{CBT} / \mathrm{MI} \text {. F/U written materials } \\
\text { upon completion. }\end{array}$ & $\begin{array}{l}\text { Not contacted after } \\
\text { multiple attempts. }\end{array}$ & $\begin{array}{l}\text { ED Utilization. Decreased. } \\
\text { Medication adherence. } \\
\text { Improved. }\end{array}$ & Moderate \\
\hline $\begin{array}{l}\text { Galloway, } \\
2011[43] \\
\text { USA, CA } \\
\text { CCT }\end{array}$ & $\begin{array}{l}\mathrm{n}=20 \text { methamphetamine-dependent } \\
\text { adults, men and women; general } \\
\text { population }\end{array}$ & $\begin{array}{l}\text { Cell phone. For } 8 \text { weeks, subjects } \\
\text { took pictures of daily morning } \\
\text { medication at time of } \\
\text { administration. Time-stamped } \\
\text { photos e'mailed to data collection } \\
\text { account. }\end{array}$ & $\begin{array}{l}\text { Medication Event } \\
\text { Monitoring System } \\
\text { (MEMS) caps on } \\
\text { bottles, weekly pill } \\
\text { counts. }\end{array}$ & $\begin{array}{l}\text { Medication adherence. } \\
\text { High levels reported. } \\
\text { Photos of medication useful } \\
\text { in measuring adherence. }\end{array}$ & Weak \\
\hline $\begin{array}{l}\text { Rosen, } 2013 \\
\text { [47] } \\
\text { USA, CA } \\
\text { RCT }\end{array}$ & $\begin{array}{l}\mathrm{n}=837(\mathrm{I}: \mathrm{n}=412, \mathrm{C}: \mathrm{n}=425) \text { veterans } \\
\text { entering residential PTSD treatment; } \\
\text { men and women, multi-site, post- } \\
\text { discharge }\end{array}$ & $\begin{array}{l}\text { Telephone. Standard outpatient } \\
\text { care plus bi-weekly phone } \\
\text { monitoring and support for } 3 \\
\text { months post-discharge. }\end{array}$ & \begin{tabular}{|l|} 
Standard outpatient \\
aftercare: referral \\
to outpatient \\
counselors, \\
psychiatrists, or \\
both.
\end{tabular} & $\begin{array}{l}\text { PTSD symptoms, } \\
\text { aggressive behaviors, } \\
\text { ETOH and drug problems, } \\
\text { depression, QOL. time to } \\
\text { rehospitalization, } \\
\text { engagement in care. No } \\
\text { significant differences. }\end{array}$ & Strong \\
\hline
\end{tabular}

Abbreviations: RCT=Randomized Controlled Trial, $\mathrm{I}=$ intervention, $\mathrm{C}=$ comparator, $\mathrm{ETOH}=\mathrm{alcohol}$, $\mathrm{BID}=$ twice daily, pharma $=$ pharmacy, $\mathrm{QOL}=$ quality of life, Dx'd=diagnosed, $\mathrm{FQHC}=$ Federally qualified health center, IVR=interactive voice response, Rx'd=prescribed, mgmt=management, $\mathrm{msg}=$ message, $\mathrm{CCT}=$ controlled clinical trial, SPMI=serious and persistent mental illness, 2nd gen=second generation, $\mathrm{CBT} / \mathrm{MI}=$ cognitive behavioral therapy/motivational interviewing, $\mathrm{F} / \mathrm{U}=$ follow up, $\mathrm{ED}=$ emergency department, $\mathrm{PTSD}=$ post-traumatic stress disorder. 
be of strong quality [31, 48], five of moderate quality [30, 32, 33, 41, 46], and three were considered of weak quality $[42,44,45]$.

Eight articles described the use of cell or smart phone technology as the study intervention [31 - 33, 41, 42, 44, 45, 48], while two did not specifically describe the type of instrument used [30,46]. The eight cell phone studies represented a variety of populations. Three articles described studies using text messaging in subjects with diabetes [32, 33, 42]; two of these were pilot studies [32, 42]. Three more pilot studies of text messaging were conducted with participants who were pregnant [41], overweight or obese [45], and in patients with hypertension [48]. One text messaging intervention was used in post-emergency department (ED) follow-up patients [31] and one pilot intervention study used IVR in healthy construction workers [44]. The two interventions that did not specify the type of phone included one study that made calls to women about mammography [30] and another using telephone IVR in individuals with diabetes [46].

Table 5. Articles that included technology use in psychiatry with non-Hispanic populations.

\begin{tabular}{|c|c|c|c|c|c|}
\hline $\begin{array}{l}\text { First Author, } \\
\text { Year } \\
\text { Location } \\
\text { Study Design }\end{array}$ & $\begin{array}{c}\text { Sample Sizes/ } \\
\text { Population/Setting }\end{array}$ & Technology/Intervention & Comparators & $\begin{array}{l}\text { Main Outcomes Intervention } \\
\text { v. Comparators }\end{array}$ & $\begin{array}{l}\text { Global } \\
\text { Quality } \\
\text { Rating }\end{array}$ \\
\hline $\begin{array}{l}\text { Allen, } 2005 \\
{[30]} \\
\text { USA, CA } \\
\text { RCT }\end{array}$ & $\begin{array}{l}\mathrm{n}=430(\mathrm{I}: \mathrm{n}=219, \mathrm{C}: \\
\mathrm{n}=211) \text { Black and } \\
\text { Hispanic women; general } \\
\text { population near } \\
\text { ambulatory care center }\end{array}$ & $\begin{array}{l}\text { Telephone. Tailored telephone } \\
\text { counseling calls to provide mammogram } \\
\text { recommendation information. }\end{array}$ & $\begin{array}{l}\text { Phone calls to } \\
\text { inquire if } \\
\text { participant had } \\
\text { screening } \\
\text { mammogram } \\
\text { since } \\
\text { enrollment. }\end{array}$ & $\begin{array}{l}\text { Screening mammogram between } \\
\text { baseline and 6-month follow-up. } \\
\text { Self-report; more screening } \\
\text { mammograms, but not } \\
\text { significant. Predictors with } \\
\text { significance: age, study group, } \\
\text { prior mammograms, knowledge } \\
\text { of age when women should } \\
\text { begin regular mammograms. }\end{array}$ & Moderate \\
\hline $\begin{array}{l}\text { Arora, } 2012 \\
\text { [32] } \\
\text { USA, CA } \\
\text { Cohort (Pilot) }\end{array}$ & $\begin{array}{l}n=23 \text { resource-poor pts } \\
\text { with diabetes, men and } \\
\text { women, Spanish or } \\
\text { English speaking; safety } \\
\text { net population }\end{array}$ & $\begin{array}{l}\text { Cell phone. Daily text messages, } \\
\text { unidirectional, English or Spanish. } \\
\text { Educational/motivational, medication } \\
\text { reminders, healthy living challenges, } \\
\text { trivia messages, phone link message to } \\
\text { allow participants to call in for free tool } \\
\text { for DM management. }\end{array}$ & None & $\begin{array}{l}\text { Healthy behaviors, DM self- } \\
\text { efficacy, medication adherence. } \\
\text { All showed improvement. } \\
\text { Diabetes knowledge. No change. } \\
\text { Satisfaction scores. Favorable. }\end{array}$ & Moderate \\
\hline $\begin{array}{l}\text { Arora, } 2014 \mathrm{a} \\
\text { [31] } \\
\text { USA, CA } \\
\text { RCT }\end{array}$ & $\begin{array}{l}\mathrm{n}=328(\mathrm{I}: \mathrm{n}=146, \mathrm{C} \\
\mathrm{n}=182) \text { low-income } \\
\text { English- and Spanish- } \\
\text { speaking pts needing } \\
\text { follow-up after ED visit }\end{array}$ & $\begin{array}{l}\text { Cell phone. Text message appointment } \\
\text { reminders, unidirectional, English or } \\
\text { Spanish. }\end{array}$ & $\begin{array}{l}\text { Usual care: } \\
\text { written follow- } \\
\text { up appointment } \\
\text { reminders. }\end{array}$ & $\begin{array}{l}\text { Post ED follow-up visits. } \\
\text { Significantly improved. }\end{array}$ & Strong \\
\hline $\begin{array}{l}\text { Arora, } 2014 \mathrm{c} \\
\text { [33] } \\
\text { USA, CA } \\
\text { RCT }\end{array}$ & $\begin{array}{l}\mathrm{n}=128(\mathrm{I}: \mathrm{n}=64, \mathrm{C}: \mathrm{n}=64) \\
\text { adult patients with poorly } \\
\text { controlled diabetes; men } \\
\text { and women, Spanish or } \\
\text { English speaking, urban, } \\
\text { public ED safety net } \\
\text { population }\end{array}$ & $\begin{array}{l}\text { ell phone. Daily text messages, } \\
\text { unidirectional, English or Spanish. In } \\
\text { addition to usual care. } \\
\text { Educational/motivational, medication } \\
\text { reminders, healthy living challenges, } \\
\text { trivia messages. }\end{array}$ & $\begin{array}{l}\text { Usual care: not } \\
\text { defined. }\end{array}$ & $\begin{array}{l}\text { HbA1C, medication adherence. } \\
\text { Improvement, but not } \\
\text { statistically significant. ED use } \\
\text { in follow-up period. Decreased. } \\
\text { Self-efficacy, self-care, diabetes } \\
\text { knowledge, QOL. Also } \\
\text { improved, not significant. ALL } \\
\text { improvements. Greater in } \\
\text { Spanish-speaking subgroup. } \\
\text { Satisfaction scores. Intervention } \\
\text { very highly rated. }\end{array}$ & Moderate \\
\hline $\begin{array}{l}\text { Evans, } 2012 \\
\text { [41] } \\
\text { USA, VA } \\
\text { RCT (Pilot) }\end{array}$ & $\begin{array}{l}\mathrm{n}=123 \text { (breakdowns } \\
\text { between I and C groups } \\
\text { unavailable) low-income } \\
\text { pregnant women, mostly } \\
\text { Hispanic; county health } \\
\text { department }\end{array}$ & $\begin{array}{l}\text { Cell phone. Text messages providing } \\
\text { prenatal education/tips + usual care. }\end{array}$ & $\begin{array}{l}\text { Usual care: } \\
\text { prenatal } \\
\text { counseling and } \\
\text { care. }\end{array}$ & $\begin{array}{l}\text { Agreement with belief in } \\
\text { preparation for motherhood. } \\
\text { Significantly improved. }\end{array}$ & Moderate \\
\hline $\begin{array}{l}\text { Fischer, } 2012 \\
\text { [42] } \\
\text { USA, CO } \\
\text { Cohort (Pilot) }\end{array}$ & $\begin{array}{l}\mathrm{n}=47 \text { low-income adult pts } \\
\text { with diabetes; English and } \\
\text { Spanish speaking; FQHC }\end{array}$ & $\begin{array}{l}\text { Cell phone. Text messages, two-way: } \\
\text { prompts for blood sugar measurement } \\
\text { and appointment reminders; pts respond } \\
\text { to both and also receive } \\
\text { acknowledgement text. English or } \\
\text { Spanish. }\end{array}$ & None & $\begin{array}{l}\text { Response to text message } \\
\text { prompts, including glucose data. } \\
\text { High rate of response. } \\
\text { Appointment attendance rates. } \\
\text { No difference from pre- } \\
\text { intervention rates. }\end{array}$ & Weak \\
\hline
\end{tabular}




\begin{tabular}{|c|c|c|c|c|c|}
\hline $\begin{array}{l}\text { First Author, } \\
\text { Year } \\
\text { Location } \\
\text { Study Design }\end{array}$ & $\begin{array}{c}\text { Sample Sizes/ } \\
\text { Population/Setting }\end{array}$ & Technology/Intervention & Comparators & $\begin{array}{c}\text { Main Outcomes Intervention } \\
\text { v. Comparators }\end{array}$ & $\begin{array}{l}\text { Global } \\
\text { Quality } \\
\text { Rating }\end{array}$ \\
\hline $\begin{array}{l}\text { Grzywacz, } 2013 \\
\text { [44] } \\
\text { USA, NC } \\
\text { Cohort (Pilot) } \\
\end{array}$ & $\begin{array}{l}\mathrm{n}=119 \text { Latino construction } \\
\text { workers; non-probability } \\
\text { sample; age } 19+; \text { general } \\
\text { population }\end{array}$ & $\begin{array}{l}\text { Cell phone IVR. VoiceXML application } \\
\text { for participant entry of daily health diary } \\
\text { information via keypad. }\end{array}$ & None & $\begin{array}{l}\text { Adherence to diary keeping. } \\
\text { Over one-third adhered; } \\
\text { feasibility supported. }\end{array}$ & Weak \\
\hline $\begin{array}{l}\text { Kolodziejczyk, } \\
2013 \text { [45] } \\
\text { USA, CA } \\
\text { Cohort (Pilot) }\end{array}$ & $\begin{array}{l}\mathrm{n}=20 \text { adult, overweight or } \\
\text { obese, English- or } \\
\text { Spanish-speaking; general } \\
\text { population }\end{array}$ & $\begin{array}{l}\text { Cell phone. Tailored daily text messages } \\
(3-5) \text { for } 8 \text { weeks }+ \text { binder w/weekly } \\
\text { weight management literature }+ \text { weekly } \\
(10-15 \text { min }) \text { counseling calls for } \\
\text { encouragement/reinforcement. English or } \\
\text { Spanish. }\end{array}$ & None & $\begin{array}{l}\text { Weight, BMI. Significant } \\
\text { decrease. Weight management } \\
\text { behaviors. Significant increase. } \\
\text { Feasibility and acceptability. } \\
\text { Favorable. }\end{array}$ & Weak \\
\hline $\begin{array}{l}\text { Lorig, } 2008 \\
\text { [46] } \\
\text { USA, CA } \\
\text { RCT }\end{array}$ & $\begin{array}{l}n=567 \text { (Study (A) I: } \\
n=219, C: n=198 \text { (to } \\
\text { reinforcement study only: } \\
n=34 ; \text { randomized to } \\
\text { reinforcement study: } \\
n=116 \text { ); Study (B) I: } \\
n=184, C: n=203 \text { (all } \\
\text { previously received } \\
\text { SDSMP)) Spanish- } \\
\text { speaking adults with type } \\
2 \text { diabetes; re-randomized } \\
\text { from first study to second } \\
\text { study; community settings }\end{array}$ & $\begin{array}{l}\text { STUDY (A) No phone. SDSMP } \\
\text { Community-based, peer-led, 6-week } \\
\text { program. STUDY (B) Telephone IVR. } \\
\text { Monthly reinforcement call: 1) } \\
\text { greeting/participant rating of ability to } \\
\text { manage DM in next month, 2) choice to } \\
\text { listen or not listen to two 90-second } \\
\text { vignettes, 3) opportunity to leave } \\
\text { message. Staff member response to } \\
\text { message, if needed. }\end{array}$ & $\begin{array}{l}\text { (A) Usual care: } \\
\text { ranging from } \\
\text { community } \\
\text { clinics to } \\
\text { specialist care; } \\
\text { (B) No } \\
\text { reinforcement } \\
\text { call. }\end{array}$ & $\begin{array}{l}\text { (A) 6-month: HbA1C, health } \\
\text { distress, hypo-/hyper-glycemia, } \\
\text { self-efficacy. All significantly } \\
\text { improved. (A) 18-month: same } \\
\text { as 6-month plus self-reported } \\
\text { global health, communication } \\
\text { w/physician. All significantly } \\
\text { improved. ALSO: physician and } \\
\text { ED visits. Decreasing trend. (B) } \\
18 \text { month: Glucose monitoring. } \\
\text { Significantly improved. } \\
\text { Otherwise, no other significant } \\
\text { differences. }\end{array}$ & Moderate \\
\hline $\begin{array}{l}\text { Sieverdes, } 2013 \\
\text { [48] } \\
\text { USA, SC } \\
\text { RCT (Pilot) }\end{array}$ & $\begin{array}{l}\mathrm{n}=10(\mathrm{I}: \mathrm{n}=5, \mathrm{C}: \mathrm{n}=5) \\
\text { adult Hispanic pts with } \\
\text { HTN, men and women } \\
\text { 50/50; FQHC }\end{array}$ & $\begin{array}{l}\text { Smart phone. SMASH app auto-collect } \\
\text { home BP (BID every } 3 \text { days) + electronic } \\
\text { med tray data (daily). Phone reminder for } \\
\text { BP, med tray (or phone if needed) } \\
\text { reminder for med. Abnormal data } \\
\text { prompted contact from study coordinator } \\
\text { or nurse. Written \& oral adherence info } \\
\text { provided to pts. Text, e'mail, or periodic } \\
\text { voice motivational/reinforcement } \\
\text { messages. }\end{array}$ & $\begin{array}{l}\text { Usual care: not } \\
\text { defined. }\end{array}$ & $\begin{array}{l}\text { Provider and pt acceptability. } \\
\text { High. Program adherence. Good } \\
\text { to excellent. Resting BP. } \\
\text { Significant improvement. } \\
\text { Ambulatory BP. Improvement } \\
\text { but not significant. }\end{array}$ & Strong \\
\hline
\end{tabular}

Abbreviations: $\mathrm{RCT}=$ randomized controlled trial, $\mathrm{I}=$ intervention, $\mathrm{C}=$ comparator, $\mathrm{DM}=$ diabetes mellitus, $\mathrm{ED}=$ emergency department, $\mathrm{HbA} 1 \mathrm{C}=$ hemoglobin $\mathrm{A} 1 \mathrm{C}, \mathrm{QOL}=$ quality of life, $\mathrm{FQHC}=$ Federally qualified health center, IVR=interactive voice response, VoiceXML=voice extensible markup language, BMI=body mass index, SDSMP=Spanish Diabetes Self-Management Program, HTN=hypertension, SMASH= Smartphone Medication Adherence Stops Hypertension, $\mathrm{BID}=$ twice daily, med=medication, $\mathrm{BP}=\mathrm{blood}$ pressure, $\mathrm{pts}=\mathrm{patients}$.

Four studies focused on diabetes management. One article described improvements in healthy behaviors, selfefficacy, and medication adherence, but no improvement in disease knowledge [32]. A second article also reported improvements in these same areas, in addition to improved clinical outcomes, disease knowledge, and QOL, most notably in the Spanish-speaking subgroup, but all lacking statistical significance [33]. A third article reported a high response rate to prompting for glucose monitoring but no improvement in appointment attendance rates [42]. The last article in the diabetes management group described two studies, the first of which did not use phone technology but provided participants for the second study that did use technology. The latter study yielded significant improvement in glucose monitoring [46]. The remaining articles discussed studies that targeted a wide range of health outcomes. In one article, increased screening mammograms were reported. Though the increase itself was not statistically significant; some predictors with significance include age, study group, prior mammograms, knowledge of when to begin getting mammograms [30]. Significant improvements were demonstrated in post-ED follow-up visits [31], health beliefs regarding preparation for motherhood [41], and weight management behaviors and clinical indicators [45]. One study showed improvements in resting and ambulatory blood pressure (BP), with significance in the former outcome measure, as well high levels of program acceptability and adherence [48]. One final article discussed demonstrated adherence to health diary keeping [44].

\section{DISCUSSION}

This systematic review identified and evaluated studies using phone technology interventions in outpatient populations published between 2003 and 2014. Specific focus was placed on Hispanic patients and psychiatric populations. Using QATQS criteria [26], six articles were assessed as strong [29, 31, 34, 40, 47, 48], eight were moderate $[30,32,33,36,37,39,41,46]$, and seven were weak in global quality [35, 38, $42-45,49]$. 
Studies included in this review demonstrate the variety of settings, patient populations, and health outcomes for which phone technology was used successfully. Eighteen studies yielded improvements in their outcome measures. Only two articles reported no improvements or equivocal results $[38,42]$ and one article reported no results at all, as it is an active study [49]. The types of phone technologies most frequently used in the articles were direct communication, in seven studies [30, 34 - 36, 39, 40, 47], text messaging, in seven studies [29, 31 - 33, 41, 42, 45], and IVR, in five studies [37, 38, 44, 46, 49]. Two other studies each used camera [43] and a smart phone application (app) [48]. Most articles specifying the use of cell phones were unclear as to type of phone, and only one study specifically mentioned the use of a smart phone intervention. Given the trend toward increasing smart phone ownership over basic cell phones [8], future studies would do well to capitalize on the myriad specialized functions of these communication devices.

\section{Phone Technology}

In studies with Hispanic participants, text messaging was the phone technology used most often, cited in six articles [31 - 33, 41, 42, 45], followed by direct communication, described in four articles [30, 34, 36, 40], IVR, in three articles [44, 46, 49], and smart phone app, in one [48]. These findings are in line with reported rates of cell phone ownership and use $[5,6]$. In contrast, studies in psychiatry favored direct communication, usually in the form of some sort of phone-based counseling or education, and cited in six studies [34 - 36, 39, 40]; IVR was used in three articles [37, 38, 49], while text intervention was discussed in only one article [29], as was cell phone camera [43]. The preference for direct communication is not surprising in a field where face-to-face meetings are the treatment norm. Though text messaging was only used in one article for psychiatry, the success of this type of intervention in Hispanics suggests that this form of technology could be helpful in other populations as well.

\section{Patient Populations}

All reviewed articles described studies conducted in outpatient populations in the U.S. except one, which took place in Ireland. Two-thirds focused on Hispanics [30 - 34, 36, 40 - 42, 44 - 46, 48, 49] and over half were in psychiatric settings or targeting psychiatric outcomes [29, 34 - 40, 43, 47, 49]. In contrast to previous reviews, this represents an increase in and, possibly, a trend toward technology research in populations where health disparities and stigma have previously been barriers to care.

\section{Health Outcomes}

The main outcomes reported by the studies in this review were quite varied, yet common threads were found among articles. The four articles that focused on psychiatric issues in Hispanics [34, 36, 40, 49] discussed depressive symptoms, QOL, and depression care, though one article did not publish results as the study was still ongoing [49]. In each of the three articles presenting results, health outcomes showed improvement with the intervention, although one article reported positive results in both the intervention and comparator groups [36].

In the seven articles discussing interventions for psychiatry in non-Hispanics [29, 35, 37 - 39, 43, 47], outcomes measured included depression and PTSD symptoms, substance use issues, medication adherence, treatment continuation, QOL, ED use, time to rehospitalization, and engagement in care. Four of these articles [29, 35, 39, 43] reported improvements in health outcomes due to the intervention. In one study medication adherence was not significantly impacted but age was determined to be a confounder [38], and in another no significant differences were noted in any of the PTSD outcomes reported [47]. Two pilot studies were included in this group and yielded good results with interventions in substance-use populations [29, 37].

The grouping of ten articles where interventions were studied for other healthcare specialties in Hispanic populations [ $30-33,41,42,44-46,48]$ described the most diverse set of outcomes, with positive results attributed to the interventions in six of the studies. Significant outcomes were reported in healthy behaviors, self-efficacy, and medication adherence [32], appointment adherence [31], health beliefs [41], weight management [45], glucose monitoring [46], and resting blood pressure [48]. One study reporting improvements in numerous outcome areas noted that all improvements were greater in the subgroup of Spanish-speakers, though none were of statistical significance [33]. This grouping of articles also contained six pilot studies [32, 41, 42, 44, 45, 48] that, in addition to reporting positive results, demonstrate acceptability and feasibility.

\section{SUMMARY}

Results from the studies conducted in Hispanic populations demonstrate that the use of mobile phone technology 
was successful and feasible across a variety of disciplines, providing evidence of positive, if not significant improvements in health outcomes. In particular, studies using text messaging interventions yielded promising results, showing that Hispanics can be targeted using this form of technology to achieve improvements in health behaviors and overall personal healthcare. A smart phone app was also shown to be a promising intervention in the Hispanic population. Overall, the literature indicates that Hispanics, as a group, are receptive and responsive to the more advanced technological features of mobile phones, and more interventions using these technologies should be undertaken.

The articles in this review also demonstrate that psychiatry, as a discipline, has not fully embraced all that technology has to offer. This is evident in the prevalence of more basic telephone and mobile modalities, such as voice and IVR, in the studies. The successful intervention among the psychiatry-focused studies using text messaging provides support for the use of more advanced mobile technology in psychiatry. Despite efforts toward reduction, a great deal of stigma surrounding mental illness remains that can, and often does, directly influence care access and continuity. The evidence shown in the literature suggests that the use of advanced mobile and smart phone technologies could provide a bridge to pharmacological and non-pharmacological treatment and appointment adherence, as well symptom reduction and improved mental well-being. Benefits realized in other disciplines, such as improvements in health attitudes, beliefs, and behaviors, including better symptom monitoring, could also be achieved in psychiatry if mobile technologies were exploited to their full capabilities.

\section{LIMITATIONS}

This review contained several limitations. The review included only English language articles and all studies but one were conducted in the U.S., which may introduce bias. Since phone technology is evolving at such a rapid pace, this review may not have captured very recent studies that may quickly be changing the state of the science. A meta-analysis was not done in this review due to the clinical diversity of the articles.

\section{CONCLUSION}

While studies have been conducted on the use of cell and smart phone interventions, few of them have focused on Hispanic patients and psychiatric populations. The articles in this review show that various types of phone technology can be helpful to patients in diverse populations. Text messaging, IVR, direct communication and smart phone app interventions have all demonstrated success in improving a variety of healthcare outcomes in these populations. These technologies can positively influence medication adherence, appointment attendance, QOL and engagement in care. Because of the high levels of ownership and generally low costs of interventions such as text messaging and smart phone apps, phone technologies can be easily adapted to numerous settings and populations and are valuable tools in efforts to increase access to care.

\section{CONFLICT OF INTEREST}

The authors confirm that this article content has no conflict of interest.

\section{ACKNOWLEDGEMENTS}

This work was supported by a Jonas Nurse Leaders Scholarship.

\section{REFERENCES}

[1] Elon University School of Communications. Imagining the Internet: A history and forecast. Available from: http://www.elon.edu/e-web/ predictions/150/1870.xhtml

[2] Kumar S, Zahn C. Mobile communications: evolution and impact on business operations. Technovation 2003; $23: 515-20$. [http://dx.doi.org/10.1016/S0166-4972(02)00120-7]

[3] Arthur C. The history of smartphone timeline Guard 2012 2012. Available from: http://www.theguardian.com/technology/2012/jan/ 24/smartphones-timeline

[4] International Telecommunication Union (ITU). The World in 2014: ICT Facts and Figures 2014. Available from: http://www. itu.int/en/ITUD/Statistics/Documents/facts/ICTFactsFigures2014-e.pdf 2014.

[5] Rainie L. Cell phone ownership hits 91\% of adults Pew Research Center 2013 2013. Available from: http://www.pewresearch.org/fact-tank/ 2013/06/06/cell-phone-ownership-hits-91-of-adults

[6] Zickuhr K, Smith A. Digital differences. Available from: http://pewinternet.org/reports/2012/digital-differences.aspx 2012. 
[7] FICO. FICO Global Survey: $80 \%$ of Smartphone Users Interested in Health Care Alerts | FICOTM Available from: http://www.fico.com/en/newsroom/fico-global-survey-80-of--smartphone-users-interested-in-health-care-alerts-06-18-2014 2014.

[8] Smith A. Smartphone Ownership 2013. Pew Res Center's Internet Am Life Proj 2013: 1-8 2013. Available from: http://www. pewinternet.og/2013/06/05/smartphone-ownership-2013/

[9] Fox S, Duggan M. Mobile Health 2012 Pew Internet Washington, DC 2012: 29 2012. Available from: http://www.pewinternet.org/ /media// Files/Reports/2012/PIP MobileHealth2012 FINAL.pdf

[10] Lopez M, Gonzalez-Barrera A, Patten E, Center P. Closing the Digital Divide: Latinos and Technology Adoption 2013. Available from: http://www.pewhispanic.org/files/2013/03/Latinos_Social_Media_and_Mobile_Tech_03-2013_final.pdf 2013.

[11] World Health Organization. WHO | Mental disorders: Fact sheet Nº396 201. Available from: http://www.who.int/mediacentre/factsheets/ fs396/en/ 2014.

[12] U.S. Department of Health and Human Services. Healthy people 2020: mental health and mental disorders 2012: 1-335 2012. Available from: http://www.healthypeople.gov/2020/topicsobjectives2020/overview.aspx?topicid=28

[13] Colom F, Vieta E, Tacchi MJ, Sánchez-Moreno J, Scott J. Identifying and improving non-adherence in bipolar disorders. Bipolar Disord 2005; 5(7 Suppl): 24-31. [http://dx.doi.org/10.1111/j.1399-5618.2005.00 248.x]

[14] Greenberg Quinlan Rosner Research Lack of medication adherence harms Americans' health: results from a US national survey of adults 2013. Available from: http://adhereforhealth.org/wp-content/uploads/pdf/2013PublicPollMemo_CAHC.pdf

[15] Mitchell AJ, Selmes T. Why don’t patients attend their appoint-ments? Maintaining engagement with psychiatric services. Adv Psychiatr Treat 2007; 13: 423-34 [http://dx.doi.org/10.1192/apt.bp.106.003202]

[16] Mitchell AJ, Selmes T. Why don't patients take their medicine? Reasons and solutions in psychiatry. Adv Psychiatr Treat 2007; 13: 336-46. [http://dx.doi.org/10.1192/apt.bp.106.003194]

[17] Nelson EA, Maruish ME, Axler JL. Effects of discharge planning and compliance with outpatient appointments on readmission rates. Psychiatr Serv 2000; 51(7): 885-9. [http://dx.doi.org/10.1176/appi.ps.51.7.885] [PMID: 10875952]

[18] Sajatovic M, Biswas K, Kilbourne AK, Fenn H, Williford W, Bauer MS. Factors associated with prospective long-term treatment adherence among individuals with bipolar disorder. Psychiatr Serv 2008; 59(7): 753-9. [http://dx.doi.org/10.1176/ps.2008.59.7.753] [PMID: 18586992]

[19] Cole-Lewis H, Kershaw T. Text messaging as a tool for behavior change in disease prevention and management. Epidemiol Rev 2010; 32: 56-69. [http://dx.doi.org/10.1093/epirev/mxq004] [PMID: 20354039]

[20] Krishna S, Boren SA, Balas EA. Healthcare via cell phones: a systematic review. Telemed J E Health 2009; 15(3): 231-40. [http://dx.doi.org/10.1089/tmj.2008.0099] [PMID: 19382860]

[21] Gurol-Urganci I, de Jongh T, Vodopivec-Jamsek V, Atun R, Car J. Mobile phone messaging reminders for attendance at healthcare appointments. Cochrane Database Syst Rev 2013; 12: CD007458. [http://dx.doi.org/10.1002/14651858.CD007458.pub3] [PMID: 24310741]

[22] Vodopivec-Jamsek V, de Jongh T, Gurol-Urganci I, Atun R, Car J. Mobile phone messaging for preventive health care. CD007457 2012. [http://dx.doi.org/10.1002/14651858.CD007457.pub2]

[23] Moher D, Liberati A, Tetzlaff J, Altman DG. Preferred reporting items for systematic reviews and meta-analyses: the PRISMA statement. Ann Intern Med 2009; 151(4): 264-269, W64. [http://dx.doi.org/10.7326/0003-4819-151-4-200908180-00135] [PMID: 19622511]

[24] Liberati A, Altman DG, Tetzlaff J, et al. The PRISMA statement for reporting systematic reviews and meta-analyses of studies that evaluate health care interventions: explanation and elaboration 2009; 62(10): e1-e34. [http://dx.doi.org/10.1016/j.jclinepi.2009.06.006]

[25] Higgins J, Green S, Eds. Cochrane Collab. Cochrane handbook for systematic reviews of interventions version 5102011 . Available from: www.cochrane-handbook.org

[26] National Collaborating Centre for Methods and Tools. Quality Assessment Tool for Quantitative Studies 2008. Available from: http://www.nccmt.ca/registry/ view/eng/14.html

[27] Thomas BH, Ciliska D, Dobbins M, Micucci S. A process for systematically reviewing the literature: providing the research evidence for public health nursing interventions. Worldviews Evid Based Nurs 2004; 1(3): 176-84.

[PMID: 17163895]

[28] Armijo-Olivo S, Stiles CR, Hagen NA, Biondo PD, Cummings GG. Hagen N a, Biondo PD, Cummings GG. Assessment of study quality for systematic reviews: a comparison of the cochrane collaboration risk of bias tool and the effective public health practice project quality assessment tool: methodological research. J Eval Clin Pract 2012; 18(1): 12-8.

[http://dx.doi.org/10.1111/j.1365-2753.2010.01516.x] [PMID: 20698919] 
[29] Agyapong VI, Ahern S, McLoughlin DM, Farren CK. Supportive text messaging for depression and comorbid alcohol use disorder: singleblind randomised trial. J Affect Disord 2012; 141(2-3): 168-76. [http://dx.doi.org/10.1016/j.jad.2012.02.040] [PMID: 22464008]

[30] Allen B Jr, Bazargan-Hejazi S. Evaluating a tailored intervention to increase screening mammography in an urban area. J Natl Med Assoc 2005; 97(10): 1350-60. [PMID: 16353657]

[31] Arora S, Burner E, Terp S, Nok Lam C, Nercisian A, Bhatt V, et al. Improving attendance at post-emergency department follow-up via automated text message appointment reminders: a randomized controlled trial. Acad Emerg Med 2014; 22 [http://dx.doi.org/10.1111/acem.12503] [PMID: 25388481]

[32] Arora S, Peters AL, Agy C, Menchine M. A mobile health intervention for inner city patients with poorly controlled diabetes: proof-ofconcept of the TExT-MED program. Diabetes Technol Ther 2012; 14(6): 492-6. [http://dx.doi.org/10.1089/dia.2011.0252] [PMID: 22524591]

[33] Arora S, Peters AL, Burner E, Lam CN, Menchine M. Trial to examine text message-based mHealth in emergency department patients with diabetes (TExT-MED): a randomized controlled trial. In: Ann Emerg Med . 2014; 62: pp. (e6)745-54. [http://dx.doi.org/10.1016/j.annemergmed.2013.10.012] [PMID: 24225332]

[34] Ashing K, Rosales M. A telephonic-based trial to reduce depressive symptoms among Latina breast cancer survivors. Psychooncology 2014; 23(5): 507-15.

[http://dx.doi.org/10.1002/pon.3441] [PMID: 24217994]

[35] Aubert RE, Fulop G, Xia F, Thiel M, Maldonato D, Woo C. Evaluation of a depression health management program to improve outcomes in first or recurrent episode depression. Am J Manag Care 2003; 9(5): 374-80. [PMID: 12744299]

[36] Badger TA, Segrin C, Hepworth JT, Pasvogel A, Weihs K, Lopez AM. Telephone-delivered health education and interpersonal counseling improve quality of life for Latinas with breast cancer and their supportive partners. Psychooncology 2013; 22(5): 1035-42. [http://dx.doi.org/10.1002/pon.3101] [PMID: 22573418]

[37] Burda C, Haack M, Duarte AC, Alemi F. Medication adherence among homeless patients: a pilot study of cell phone effectiveness. J Am Acad Nurse Pract 2012; 24(11): 675-81. [http://dx.doi.org/10.1111/j.1745-7599.2012.00756.x] [PMID: 23088699]

[38] Castle T, Cunningham MA, Marsh GM. Antidepressant medication adherence via interactive voice response telephone calls. Am J Manag Care 2012; 18(9): e346-55. [PMID: 23009333]

[39] Cook PF, Emiliozzi S, Waters C, El Hajj D. Effects of telephone counseling on antipsychotic adherence and emergency department utilization. Am J Manag Care 2008; 14(12): 841-6. [PMID: 19067501]

[40] Ell K, Katon W, Xie B, et al. One-year postcollaborative depression care trial outcomes among predominantly Hispanic diabetes safety net patients 2011. [http://dx.doi.org/10.1016/j.genhosppsych.2011.05.018]

[41] Evans WD, Wallace JL, Snider J. Pilot evaluation of the text4baby mobile health program. BMC Public Health 2012; 12: 1031-40. [http://dx.doi.org/10.1186/1471-2458-12-1031] [PMID: 23181985]

[42] Fischer HH, Moore SL, Ginosar D, et al. Care by cell phone: text messaging for chronic disease management. Am J Manag Care 2012; 18(2): e42-7. [PMID: 22435883]

[43] Galloway GP, Coyle JR, Guillén JE, Flower K, Mendelson JE. A simple, novel method for assessing medication adherence: capsule photographs taken with cellular telephones. J Addict Med 2011; 5(3): 170-4. [http://dx.doi.org/10.1097/ADM.0b013e3181fcb5fd] [PMID: 21844832]

[44] Grzywacz JG, Quandt SA, Marín A, et al. Feasibility of interactive voice response methods in health behavior research with immigrant Latinos 2013. [http://dx.doi.org/10.5993/AJHB.37.2.11]

[45] Kolodziejczyk JK, Norman GJ, Barrera-Ng A, et al. Feasibility and effectiveness of an automated bilingual text message intervention for weight loss: pilot study. J Med Internet Res Protoc 2013; 2(2): e48. [http://dx.doi.org/10.2196/resprot.2789] [PMID: 24200517]

[46] Lorig K, Ritter PL, Villa F, Piette JD. Spanish diabetes self-management with and without automated telephone reinforcement: two randomized trials. Diabetes Care 2008; 31(3): 408-14. [http://dx.doi.org/10.2337/dc07-1313] [PMID: 18096810]

[47] Rosen CS, Tiet QQ, Harris AH, et al. Telephone monitoring and support after discharge from residential PTSD treatment: a randomized controlled trial. Psychiatr Serv 2013; 64(1): 13-20. [http://dx.doi.org/10.1176/appi.ps.201200142] [PMID: 23117443]

[48] Sieverdes J, Patel S, Davidson L, Treiber F. mHealth medication and blood pressure self-management program in Hispanic hypertensives: a 
proof of concept trial. Smart Homecare Technol TeleHealth 2013; 2: 1 .

[http://dx.doi.org/10.2147/SHTT.S49633]

[49] Wu S, Ell K, Gross-Schulman SG, et al. Technology-facilitated depression care management among predominantly Latino diabetes patients within a public safety net care system: comparative effectiveness trial design 2014; 37: 342-54.

[http://dx.doi.org/10.1016/j.cct.2013.11.002]

(C) Duarte and Thomas; Licensee Bentham Open.

This is an open access article licensed under the terms of the Creative Commons Attribution-Non-Commercial 4.0 International Public License (CC BY-NC 4.0) (https://creativecommons.org/licenses/by-nc/4.0/legalcode), which permits unrestricted, non-commercial use, distribution and reproduction in any medium, provided the work is properly cited. 